and $69 \%, 89 \%, 77 \%, 91 \%, 64 \%$ for 3D perfusion CMR (AUC of 0.88 versus $0.79, \mathrm{p}=0.27$ ). In 24 patients with confirmed $\mathrm{CAD}$, the $\mathrm{MIB}$ by $2 \mathrm{D}$ and $3 \mathrm{D}$ was $15.7 \pm 8.6 \%$ and $19.6 \% \pm 11.7 \%$ respectively $(\mathrm{p}=0.088)$, with a trend towards $3 \mathrm{D}$ underestimating $\mathrm{MIB}$ by a mean of $3.8 \%$ (Figure 1 ). In these patients, considering $2 \mathrm{D}$ as the gold standard, the diagnostic accuracy of 3D CMR, anatomical BCIS-JS, and functional BCIS-JS in identifying prognostically significant MIB was $79 \%, 75 \%$, and $87.5 \%$ respectively. (See Figures 2 and 3 for case examples)

Conclusion In this first head-to-head comparison with invasive angiography and FFR, high-resolution 2D and whole-heart 3D perfusion CMR had comparable diagnostic performance in detecting flow-limiting CAD on a per-patient basis. Both 3D and functional BCIS-JS identify prognostically significant MIB well as determined by 2D. 2D estimates of MIB tend to be higher than 3D, however both methods have limitations (resolution versus coverage). In this contest, superior resolution may satisfactorily offset the lack of myocardial coverage.

\section{NOVEL PERFUSION CMR REFERENCE STANDARD FOR THE OBJECTIVE DIAGNOSIS OF MICROCIRCULATORY DYSFUNCTION - VALIDATION AGAINST PROGNOSTIC INVASIVE MARKERS OF CORONARY PHYSIOLOGY}

Alexander Liu, Rohan S Wijesurendra, Joanna M Liu, John C Forfar, Keith M Channon, Stefan K Piechnik, Stefan Neubauer, Rajesh K Kharbanda*, Vanessa M Ferreira . University of Oxford, John Raddliffe Hospital, Oxford, UK; * These senior authors contributed equally

\subsection{6/heartjnl-2017-311399.22}

Objectives In patients with angina and non-obstructive coronary arteries, abnormal index of microcirculatory resistance (IMR $\geq 23$ ) and coronary flow reserve (CFR $\leq 2.0$ ) confer adverse clinical outcomes. We hypothesised that these prognostic invasive markers of microcirculatory dysfunction are related to impaired downstream myocardial perfusion reserve (MPR) as assessed by CMR.

Methods 60 subjects (20 patients with angina and non-obstructive coronary arteries, 20 patients with obstructive coronary

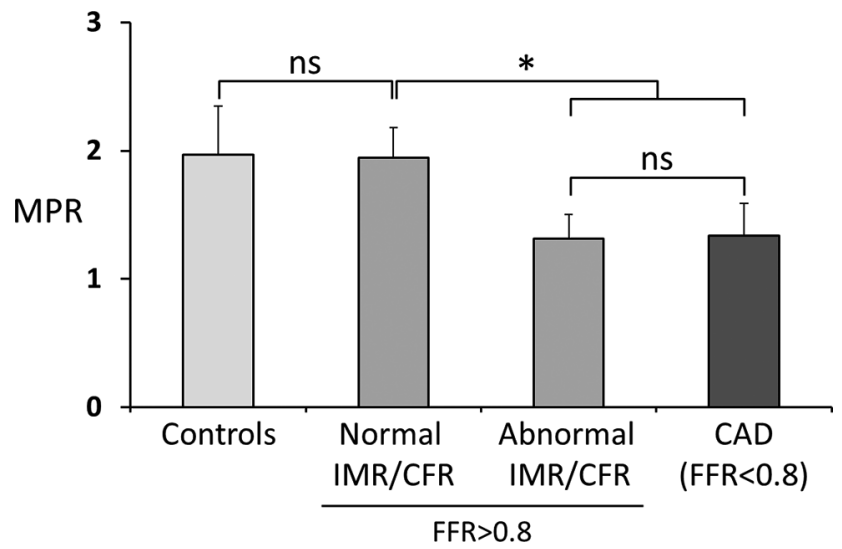

Abstract 022 Figure 1 Patterns of myocardial perfusion reserve (MPR) in normal controls, and downstream of non-obstructive coronary arteries, and obstructive coronary arteries (CAD). All bars represent mean $\pm S D,{ }^{*} p<0.05$, ns denotes $p>0.10$. IMR: index of

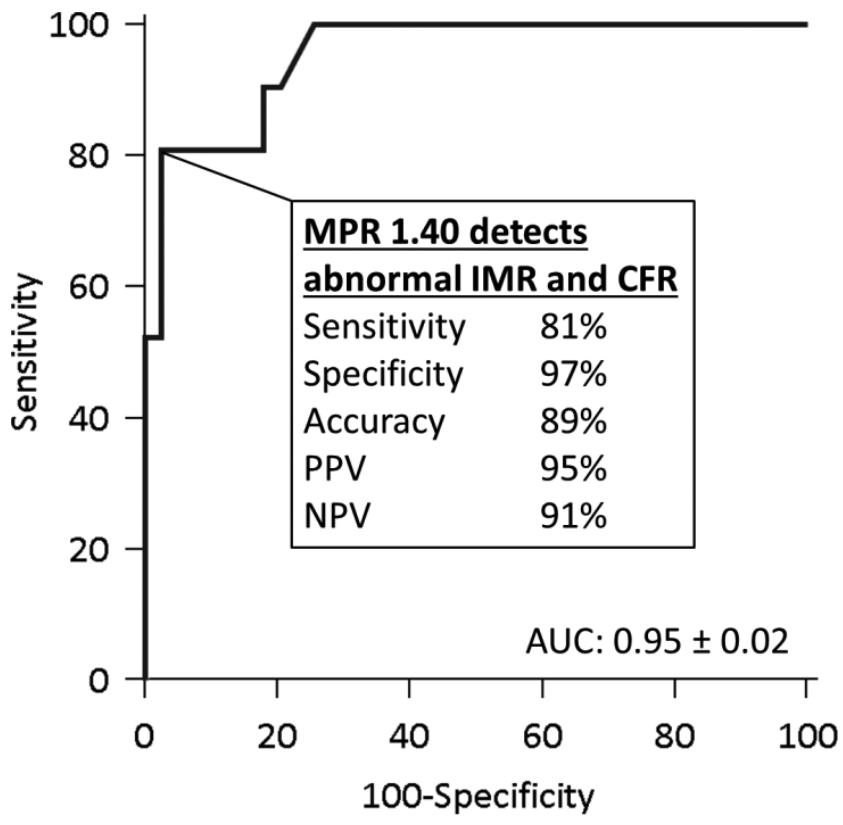

Abstract 022 Figure 2 Diagnostic performance of myocardial perfusion reserve (MPR) for diagnosing microcirculatory dysfunction (true positives: abnormal IMR $\geq 23$ and $\mathrm{CFR} \leq 2.0$; true negatives: IMR $<23$ and $C F R>2.0$ ). AUC: area under the curve.

artery disease [CAD] and 20 normal controls) underwent CMR for the assessment of LV function (cines), ischaemia (adenosine stress and rest perfusion) and infarction (LGE). During invasive coronary angiography, patients with nonobstructive coronary arteries had fractional flow reserve (FFR), CFR and IMR measured in all 3 coronary arteries (60 vessels), while patients with obstructive CAD had FFR measured in the obstructive coronary artery only (20 vessels). CMR images were analysed blinded to clinical information and invasive coronary data. MPR was derived as the ratio between stress and rest myocardial perfusion signal intensity upslope gradients, normalised to LV blood signal intensity.

Results Myocardium with LGE was excluded. Non-infarcted myocardium downstream obstructive coronary arteries (FFR $<0.80)$ had lower MPR compared to controls $(1.3 \pm 0.4$ vs 1.9 $\pm 0.3, \quad \mathrm{p}<0.001)$. Downstream of non-obstructive coronary arteries (FFR $>0.80$ ), myocardium with normal microcirculatory function (normal IMR and CFR) had similar MPR compared to controls $(1.9 \pm 0.2$ vs $1.9 \pm 0.3, p=0.83)$. Myocardium with prognostic microcirculatory dysfunction (IMR $\geq 23$ and CFR $\leq 2.0)$ had significantly impaired MPR (1.3 \pm 0.3 , $\mathrm{p}<0.001)$; further, the degree of impairment in MPR was similar to ischaemic myocardium downstream obstructive coronary arteries (MPR $1.3 \pm 0.3$ vs $1.3 \pm 0.4, p=0.73$; Figure 1 ). On ROC analysis, a threshold of $\mathrm{MPR}=1.4$ detected prognostic microvascular dysfunction (IMR $\geq 23$ and CFR $\leq 2.0$ ) in nonobstructive coronary arteries with specificity 97\%, sensitivity $81 \%$, and accuracy 89\% (AUC 0.95 \pm 0.02 , Figure 2).

Conclusions In patients with angina and non-obstructive coronary arteries, an MPR threshold of 1.4 on perfusion CMR accurately detects prognostic invasive markers of microcirculatory dysfunction. This novel MPR threshold can now be used to objectively diagnose microvascular angina in clinical practice and guide disease management. 